\title{
TRANSIENT MOMENTS OF THE TCP WINDOW SIZE PROCESS
}

\author{
ANDREAS H. LÖPKER, ${ }^{* * *}$ EURANDOM \\ JOHAN S. H. VAN LEEUWAARDEN, ${ }^{* * *}$ EURANDOM and Eindhoven University of Technology
}

\begin{abstract}
The TCP window size process can be modeled as a piecewise-deterministic Markov process that increases linearly and experiences downward jumps at Poisson times. We present a transient analysis of this window size process. Our main result is the Laplace transform of the transient moments. Formulae for the integer and fractional moments are derived, as well as an explicit characterization of the speed of convergence to steady state. Central to our approach are the infinitesimal generator and Dynkin's martingale.
\end{abstract}

Keywords: Piecewise-deterministic Markov process; Dynkin's martingale; optional stopping; TCP; window size; AIMD; stationary distribution; transient moments; exponential functional; difference equation; mixing time

2000 Mathematics Subject Classification: Primary 60J25; 60J35; 60K10; 90B20; 90B15

Secondary 90B18; 60K30; 60G44; 68M20

\section{Introduction}

Data transfer over the Internet is predominantly controlled by the transmission control protocol (TCP), which adapts the window size (transmission rate) of data transfers to the congestion of the network. A TCP connection between a source and a destination progressively increases the window size, until it receives a signal that its path in the network is too congested, upon which the window size is drastically reduced. The most common implementation of TCP uses an additive-increase, multiplicative-decrease (AIMD) algorithm. This allows the window size to increase linearly in the absence of congestion signals, whereas when congestion is detected, the window size is reduced by a multiplicative factor.

The emergence of TCP has spurred an enormous amount of research. In the pioneering work of Ott et al. [18], the window size process is modeled as a piecewise-deterministic Markov process (PDMP). Our framework incorporates the model in [18], as well as some of the extensions made in [1], [2], and [13], and is closely related to the models in [4] and [5]. All these works restrict to the stationary behavior of the process, which is tantamount to the assumption that the TCP connection is persistent enough so that its throughput is governed by the stationary regime. We obtain results on the transient moments of the PDMP. Our results may help in judging the effects that parameters have on the dynamics of the system.

We model the window size as a Markov process $\left(X_{t}\right)_{t \geq 0}$ that increases linearly with rate 1 . Congestion signals arrive according to a Poisson process with rate $\lambda$, and upon receipt of the $i$ th signal, the window size is reduced by multiplication with a random variable $Q_{i}$. We assume

Received 28 March 2007; revision received 24 January 2008.

* Postal address: EURANDOM, PO Box 513, 5600 MB Eindhoven, The Netherlands.

** Email address: lopker@eurandom.tue.nl

*** Email address: j.s.h.v.leeuwaarden@tue.nl

Supported by an NWO Veni grant. 
that $\left(Q_{i}\right)_{i \in \mathbb{N}}$ is a sequence of independent and identically distributed (i.i.d.) random variables with range $[0,1)$. Let $Q$ denote a generic random variable equal in distribution to $Q_{i}$. TCP corresponds to the special case in which $Q=q$ for some constant $q$ in $[0,1)$, with $q=\frac{1}{2}$ being the most common choice.

We obtain all transient moments of the window size for any possible starting point $X_{0}=x$. The main mathematical technique we use stems from the field of PDMPs and involves the analysis of the infinitesimal generator using Dynkin's formula.

A quantity of particular interest is the relaxation time (sometimes referred to as the mixing time), loosely defined as the time it takes for the PDMP to reach stationarity. Being in possession of the explicit formulae for the transient moments, we can measure the relaxation time in terms of the difference between the transient and the stationary moments. Let $\mathrm{E}_{x}\left(X_{t}^{n}\right)$ denote the $n$th moment of the Markov process at time $t$ with $X_{0}=x$. We find that (see Theorem 4)

$$
\mathrm{E}_{x}\left(X_{t}^{n}\right)=\mathrm{E}\left(X_{\infty}^{n}\right)+\sum_{k=1}^{n} c_{k, n, x} \exp \left(-\theta_{k} t\right)
$$

with $\theta_{k}=\lambda\left(1-\mathrm{E}\left(Q^{k}\right)\right)$, and where both $\mathrm{E}\left(X_{\infty}^{n}\right)$ and $c_{k, n, x}$ are fully expressed in terms of $\theta_{1}, \ldots, \theta_{n}$. From (1) we see that there is an exponential speed of convergence to the stationary moments. The relaxation time can be defined as the time until the difference between $\mathrm{E}_{x}\left(X_{t}^{n}\right)$ and its stationary counterpart $\mathrm{E}\left(X_{\infty}^{n}\right)$ is smaller than some predetermined value. Ott and Kemperman [17] recently obtained the transient moments for the case in which $Q=q$. Our Theorem 4 is in agreement with [17, Equation (3.10)].

There are some connections to other fields. First, our PDMP is part of a larger class of models known as growth-collapse processes, which are real-valued processes that grow between random collapse times, at which they jump down according to some distribution depending on their current level. This evolutionary pattern is encountered in a large variety of physical phenomena (see [10]) such as the buildup of friction, earthquakes, avalanches, neuron firing, and shot noise. Insurance mathematics [20], inventory theory [21], and queueing theory [3] are other fields where growth rate and occasional disasters are witnessed and analyzed. There is a second connection to the field of stochastic recursive equations of the type

$$
X \stackrel{\mathrm{D}}{=} Q X+Z
$$

where $X, Q$, and $Z$ are random variables, $X$ is independent of $Q$ and $Z$, and ' $=$, denotes equality in distribution. Indeed, the limiting random variable $X_{\infty}$ of our Markov process satisfies such an equation. Vervaat [22] provided a detailed study of these equations and several examples of explicit solutions for particular choices of $(Q, Z)$ (see also [11]). A third connection is shown in Section 6. As it turns out, our Markov process is equal in distribution to the exponential functional associated to a Lévy (compound Poisson) process.

The paper is structured as follows. In Section 2 we give a detailed model description. The infinitesimal generator of the PDMP plays a fundamental role in our approach. We prove that a certain class of nonlocally bounded functions belongs to the domain of the generator. In Section 3 we investigate the stationary distribution of the Markov process, making use only of the generator. Section 4 presents the transient analysis of the Markov process and comprises the core of this paper. We start with a derivation of the Laplace transform of the transient moments. The proof uses the generator, Dynkin's martingale, the solution of an inhomogeneous linear difference equation, and the Bohr-Mollerup theorem. The Laplace transform is then shown to lead to fractional and integer moments of the stationary and transient distribution. A brief 
discussion follows in Section 5. We conclude the paper with the connection to Lévy processes in Section 6.

\section{Model description}

Consider a Markov process $\left(X_{t}\right)_{t \geq 0}$ that increases with slope 1 and has random jumps at Poisson times $\left(T_{i}\right)_{i \in \mathbb{N}}$; see Figure 1. The $i$ th jump goes from $X_{T_{i}-}$ to $X_{T_{i}}=Q_{i} X_{T_{i}-}$, where $\left(Q_{i}\right)_{i \in \mathbb{N}}$ is a sequence of i.i.d. random variables with range $[0,1)$ and probability distribution function $H$. Let $\lambda$ be the intensity of the Poisson process, and let $N_{t}$ count the number of jumps in $[0, t]$. Moreover, let $Q$ denote a generic random variable with distribution function $H$, and let

$$
\theta_{a}=\lambda\left(1-\mathrm{E}\left(Q^{a}\right)\right)
$$

for $a>a_{\min }=\inf _{c \in \mathbb{R}}\left\{\mathrm{E}\left(Q^{c}\right)<\infty\right\}$. It turns out that these quantities are crucial for the description of the transient behavior of $\left(X_{t}\right)_{t \geq 0}$. A connection between $\theta_{a}$ and the Laplace exponent of an associated Lévy process is given in Section 6.

The process $\left(X_{t}\right)_{t \geq 0}$ is an example of a PDMP introduced in [9]. The state space $\mathbb{S}$ consists of all nonnegative real numbers; thus, $\mathbb{S}=[0, \infty)$. If no jumps to 0 occur then we agree to set $\mathbb{S}=(0, \infty)$.

The full infinitesimal generator of the Markov process $\left(X_{t}\right)_{t \geq 0}$ is given by

$$
\mathcal{A} f(x)=f^{\prime}(x)-\lambda f(x)+\lambda \int_{0}^{1} f(y x) \mathrm{d} H(y), \quad x \in \mathbb{S},
$$

where $f^{\prime}$ denotes the density of the absolutely continuous function $f$. The domain of the generator consists of all measurable functions $f: \mathbb{S} \rightarrow \mathbb{R}$ for which the process $f\left(X_{t}\right)-\int_{0}^{t} \mathcal{A} f\left(X_{S}\right) \mathrm{d} s$ is a martingale. According to Davis [9], a function $f: \mathbb{S} \rightarrow \mathbb{R}$ belongs to the domain of $\mathcal{A}$ if it is absolutely continuous on $\mathbb{S}$ and the expectation of $\sum_{k=1}^{N_{t}}\left|f\left(X_{T_{k}-}\right)-f\left(X_{T_{k}}\right)\right|$ is finite for every choice of $t \geq 0$ and $x>0$. This is the case, for example, if $f$ is absolutely continuous and locally bounded on $\mathbb{S}$, and this subclass will be sufficiently rich in most cases. However, since we also deal with certain nonlocally bounded functions, we show the following result, which identifies a subclass of the domain of $\mathscr{A}$ that contains functions like the negative powers $x^{a}, a<0$.

Lemma 1. Let $f: \mathbb{S} \rightarrow[0, \infty)$ be a nonincreasing function with $f(x y) \leq f(x) f(y)$ for all $x, y \in \mathbb{S}$. Then $f$ belongs to the domain of the generator if $\mathrm{E}_{x} f(Q)<\infty$ for all $x \in \mathbb{S}$.

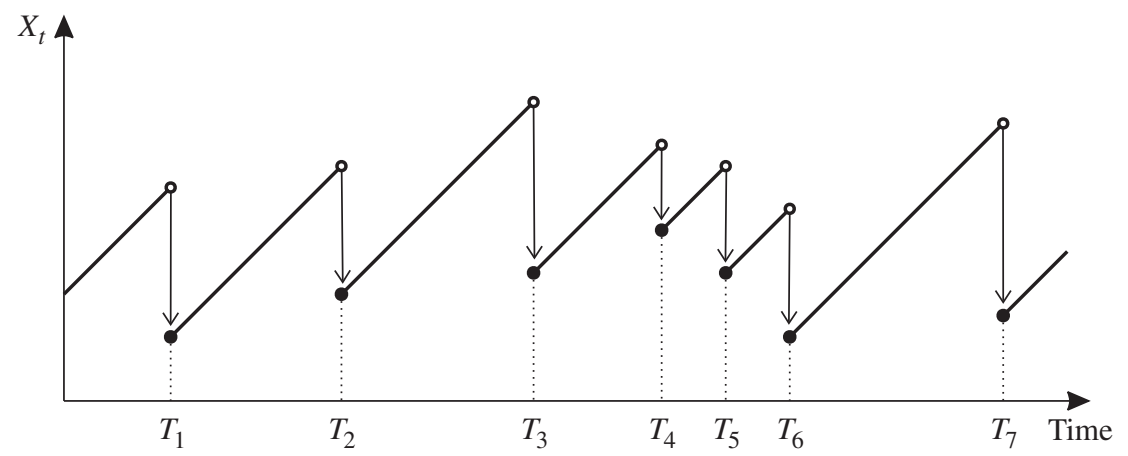

Figure 1: The process $\left(X_{t}\right)_{t \geq 0}$. Linear increase with slope 1 and random jumps at Poisson times $\left(T_{i}\right)_{i \in \mathbb{N}}$. The $i$ th jump goes from $X_{T_{i}-}$ to $X_{T_{i}}=Q_{i} X_{T_{i}-}$. 
Proof. Let $M_{t}=\min _{s \in\left[T_{1} \wedge t, t\right]} X_{s}$. For $k \leq N_{t}$ and, thus, $T_{k} \leq t$,

$$
\left|f\left(X_{T_{k}-}\right)-f\left(X_{T_{k}}\right)\right| \leq f\left(X_{T_{k}-}\right)+f\left(X_{T_{k}}\right) \leq 2 f\left(X_{T_{k}}\right) \leq 2 f\left(M_{t}\right),
$$

which gives

$$
\mathrm{E}_{x}\left(\sum_{k=1}^{N_{t}}\left|f\left(X_{T_{k}-}\right)-f\left(X_{T_{k}}\right)\right|\right) \leq 2 \mathrm{E}_{x}\left(f\left(M_{t}\right) N_{t}\right) .
$$

Clearly, $M_{t}=X_{T_{k}}$ for some $0 \leq k \leq N_{t}$, if we let $T_{0}=0$. Then

$$
M_{t}=\left(\cdots\left(\left(X_{0}+Z_{1}\right) Q_{1}+Z_{2}\right) Q_{2}+\cdots\right) Q_{k},
$$

where $\left(Z_{k}\right)_{k \geq 1}$ are independent and exponentially distributed random variables. So, $M_{t} \geq$ $X_{\left(t \wedge T_{1}\right)-W_{t}}$, where $W_{t}=Q_{1} Q_{2} \cdots Q_{N_{t}}$. Consequently, using the monotonicity of $f$,

$$
\mathrm{E}_{x}\left(f\left(M_{t}\right) N_{t}\right) \leq \mathrm{E}_{x}\left(f \left(X_{\left.\left.\left(t \wedge T_{1}\right)-W_{t}\right) N_{t}\right)}\right.\right.
$$

Conditioning on the first jump time $T_{1}$ yields

$$
\begin{aligned}
\mathrm{E}_{x}\left(f\left(X_{t \wedge T_{1}-} W_{t}\right) N_{t}\right) & =\int_{0}^{\infty} \lambda \mathrm{e}^{-\lambda y} \mathrm{E}_{x}\left(f\left(X_{(t \wedge y)-} W_{t}\right) N_{t} \mid T_{1}=y\right) \mathrm{d} y \\
& =\int_{0}^{t} \lambda \mathrm{e}^{-\lambda y} \mathrm{E}_{x}\left(f\left((x+y) W_{t-y}\right) N_{t-y}\right) \mathrm{d} y .
\end{aligned}
$$

Next we condition on $N_{t-y}$ to obtain

$$
\begin{aligned}
\int_{0}^{t} \lambda \mathrm{e}^{-\lambda y} \mathrm{E}_{x}\left(f\left((x+y) W_{t-y}\right) N_{t-y}\right) \mathrm{d} y \\
\quad=\int_{0}^{t} \sum_{n=0}^{\infty} n \mathrm{P}\left(N_{t-y}=n\right) \lambda \mathrm{e}^{-\lambda y} \mathrm{E}_{x}\left(f\left((x+y) Q_{1} \cdots Q_{n}\right)\right) \mathrm{d} y \\
\quad \leq \int_{0}^{t} \sum_{n=0}^{\infty} n \mathrm{P}\left(N_{t-y}=n\right) \mathrm{E}_{x} f(Q)^{n} \lambda \mathrm{e}^{-\lambda y} f(x+y) \mathrm{d} y,
\end{aligned}
$$

which is clearly finite for all $x>0$ and all $t \geq 0$ as long as $\mathrm{E}_{x} f(Q)<\infty$.

\section{Stationary behavior}

Since, for large values of $X_{t}$, the downward jumps always dominate the deterministic linear increase and since the jump intensity $\lambda$ is constant, it is plausible that the process will always be stable, in the sense that a limiting distribution for $X_{t}$ exists as $t$ tends to $\infty$. That this is actually the case is established in the following theorem.

Theorem 1. The process $\left(X_{t}\right)_{t \geq 0}$ always has a stationary distribution.

Proof. Let $z=(1+\delta) / \theta_{1}$, and let $\tau_{z}=\inf \left\{t>0 \mid X_{t}=z\right\}$. We first show that the mean of $\tau_{z}$ is finite if we start the process in $x \leq z$. Let $\tilde{X}_{t}$ be a process with the same deterministic behavior and the same jump times $T_{i}$ as $X_{t}$ but with $X_{0}=0$ and jumps that always go to 0 . If we show that the expectation of $\tilde{\tau}_{z}=\inf \left\{t>0 \mid \tilde{X}_{t}=z\right\}$ is finite, the same follows for $\tau_{z}$, since $\tilde{X}_{t}$ always stays below $X_{t}$. Let $N$ be the number of jumps before $\tilde{\tau}_{z}$. Then $N$ has 
a geometric distribution with parameter $\mathrm{e}^{-\lambda z}$ and $\tilde{\tau}_{z}$ is a geometric sum of random variables, which is bounded by $z$. It follows that $\mathrm{E}_{0} \tilde{\tau}_{z}<\infty$ and, hence, $\mathrm{E}_{x} \tau_{z}<\infty$.

Next we start the process $X_{t}$ in $x \geq z$ and again show that $\mathrm{E}_{x} \tau_{z}<\infty$. Let $p_{1}(x)=x$. Then $p_{1}$ is in the domain of $\mathcal{A}$ and $\mathcal{A} p_{1}(x)=1-\theta_{1} x$.

Choose a $\delta>0$, and let $\tau=\inf \left\{t>0 \mid X_{t} \leq z\right\}$. Up to time $\tau$ the process $X_{t}+$ $\theta_{1} \int_{0}^{t} X_{s} \mathrm{~d} s-t$ is a supermartingale, bounded below by $z+\delta t>0$. It follows that

$$
f(x) \geq \mathrm{E}_{x}\left(X_{\tau}+\theta_{1} \int_{0}^{\tau} X_{s} \mathrm{~d} s-\tau\right) \geq \frac{1+\delta}{\theta_{1}}+\delta \mathrm{E}_{x}(\tau),
$$

implying that $\mathrm{E}_{x} \tau<\infty$. Hence, the expected time the process needs to go from $z$ back to $z$, which is $\mathrm{E}_{z} \tau_{z}+\mathrm{E}\left(\mathrm{E}_{X_{\tau_{z}}} \tau\right)$, is finite. From the theory of regenerative processes, it follows that $\left(X_{t}\right)_{t \geq 0}$ has a stationary distribution (cf. [3]).

Theorem 2. The density $v^{\prime}$ of the stationary distribution $v$ satisfies the equation

$$
v^{\prime}(z)=\lambda\left(\int_{0}^{1} v\left(\frac{z}{y}\right) \mathrm{d} H(y)-v(z)\right) .
$$

If $\psi(s)=\int_{0}^{\infty} \mathrm{e}^{-s t} \mathrm{~d} v(t)$ denotes the Laplace transform of $v$ then

$$
\psi(s)=\frac{\lambda}{\lambda+s} \int_{0}^{1} \psi(s y) \mathrm{d} H(y) .
$$

Moreover, at least for $0 \leq s \leq \lambda$,

$$
\psi(s)=\sum_{n=0}^{\infty} \frac{(-s)^{n}}{\prod_{k=1}^{n} \theta_{k}} .
$$

Proof. We first consider the Laplace transform $\psi(s)$. The function $f(x)=\mathrm{e}^{-s x}$ is a bounded member of the domain of $\mathcal{A}$ and

$$
\mathcal{A} f(x)=-(\lambda+s) \mathrm{e}^{-s x}+\lambda \int_{0}^{1} \mathrm{e}^{-s y x} \mathrm{~d} H(y),
$$

so that (4) follows by integrating with respect to $v$ since, for all bounded $f$ in the domain of $\mathcal{A}$, we have $\int_{0}^{\infty} \mathcal{A} f(x) \mathrm{d} v(x)=0$. Equation (3) follows immediately by inversion.

Since $\lim \inf \theta_{k} \leq \lambda$ as $k \rightarrow \infty$, the radius of convergence of the series in (5) is clearly larger than $\lambda$. By inserting (5) into (4) we find that

$$
\begin{aligned}
\frac{\lambda}{\lambda+s} \int_{0}^{1} \sum_{n=0}^{\infty} \frac{(-s y)^{n}}{\prod_{k=1}^{n} \theta_{k}} \mathrm{~d} H(y) & =\frac{\lambda}{\lambda+s} \sum_{n=0}^{\infty} \frac{(-s)^{n} \int_{0}^{1} y^{n} \mathrm{~d} H(y)}{\prod_{k=1}^{n} \theta_{k}} \\
& =\frac{\lambda}{\lambda+s} \sum_{n=0}^{\infty} \frac{(-s)^{n}\left(1-\theta_{n} / \lambda\right)}{\prod_{k=1}^{n} \theta_{k}} \\
& =\frac{\lambda}{\lambda+s}\left(\sum_{n=0}^{\infty} \frac{(-s)^{n}}{\prod_{k=1}^{n} \theta_{k}}+\frac{s}{\lambda} \sum_{n=0}^{\infty} \frac{(-s)^{n-1}}{\prod_{k=1}^{n-1} \theta_{k}}\right) \\
& =\sum_{n=0}^{\infty} \frac{(-s)^{n}}{\prod_{k=1}^{n} \theta_{k}},
\end{aligned}
$$

and, hence, the series in (5) actually represents $\psi$. 
The results of Theorem 2 are not new. In fact, the Laplace transform of the stationary distribution has been derived in Guillemin et al. [13], although both the setting and the method of proof is different from ours. They considered the same process, but represented our random variable $Q$ as $q^{R}$ with $q \in[0,1)$ and $R$ some nonnegative random variable. Guillemin et al. [13] considered the stochastic recursive equation

$$
X_{\infty} \stackrel{\mathrm{D}}{=} Q X_{\infty}+Z
$$

where $X_{\infty}, Q$, and $Z$ are independent, and $Z$ is exponential with mean $1 / \lambda$. It then follows that

$$
\psi(s)=\mathrm{Ee}^{-s Z} \mathrm{E} \exp \left(-s Q X_{\infty}\right)=\mathrm{Ee}^{-s Z} \int_{0}^{1} \mathrm{E} \exp \left(-s y X_{\infty}\right) \mathrm{d} H(y),
$$

yielding (4). Also, for $n \in \mathbb{N}$,

$$
\mathrm{E}\left(X_{\infty}^{n}\right)=\mathrm{E}\left(\left(Q X_{\infty}+Z\right)^{n}\right)=\sum_{k=0}^{n}\left(\begin{array}{l}
n \\
k
\end{array}\right) \mathrm{E}\left(Q^{k}\right) \mathrm{E}\left(X_{\infty}^{k}\right) \mathrm{E}\left(Z^{n-k}\right)
$$

(see also [12, p. 481]), which gives

$$
\mathrm{E}\left(X_{\infty}^{n}\right)=\frac{n !}{\lambda^{n} \theta_{n}} \sum_{k=0}^{n-1}\left(\lambda-\theta_{k}\right) \frac{\lambda^{k}}{k !} \mathrm{E}\left(X_{\infty}^{k}\right)=\frac{n !}{\prod_{i=1}^{n} \theta_{i}} .
$$

These integer moments, with $\psi(s)=\sum_{n=0}^{\infty} \mathrm{E}\left(X_{\infty}^{n}\right)(-s)^{n} / n$ ! and after checking Carleman's criterion, then lead to (5); see Proposition 8 of [13].

We point out that our proof of Theorem 2 strongly builds on the properties of the infinitesimal generator, an approach that will prove its value in the upcoming section on transient analysis.

\section{Transient moments}

We now derive a formula for the Laplace transform of the transient moments. More precisely, Theorem 3, below, provides a formula for

$$
\mu_{x}^{a}(u)=\int_{0}^{\infty} \mathrm{e}^{-u t} \mathrm{E}_{x}\left(X_{t}^{a}\right) \mathrm{d} t .
$$

We start by observing that this function satisfies a certain difference equation in $a$.

Lemma 2. If $a>a_{\min }$ and $-a \notin \mathbb{N} \cup\{0\}$ then $\mathrm{E}_{x}\left(X_{t}^{a-1}\right)<\infty$ and

$$
\mu_{x}^{a}(u)=\frac{x^{a}+a \mu_{x}^{a-1}(u)}{\theta_{a}+u} .
$$

Proof. The function $p_{a}(x)=x^{a}$ is absolutely continuous and if $\mathrm{E}\left(Q^{a}\right)<\infty$ then $p_{a}$ satisfies the conditions of Lemma 1 . Indeed, $p_{a}$ is nonincreasing and $p_{a}(x y)=p_{a}(x) p_{a}(y)$. Consequently, $p_{a}$ is in the domain of the generator. We have, from (2) with $f(x)=p_{a}(x)$,

$$
\mathcal{A} p_{a}(x)=a x^{a-1}-x^{a} \int_{0}^{1} \lambda\left(1-y^{a}\right) \mathrm{d} H(y)=a x^{a-1}-\theta_{a} x^{a} .
$$


Then, by Dynkin's formula (cf. [9, Proposition 14.13]),

$$
p_{a}\left(X_{t}\right)-\int_{0}^{t} \mathcal{A} p_{a}\left(X_{s}\right) \mathrm{d} s=X_{t}^{a}-\int_{0}^{t} a X_{s}^{a-1} \mathrm{~d} s-\theta_{a} \int_{0}^{t} X_{s}^{a} \mathrm{~d} s
$$

is a martingale. In particular, its mean is constantly equal to $\mathrm{E}_{x} X_{0}^{a}=x^{a}$; thus,

$$
\mathrm{E}_{x}\left(X_{t}^{a}-\int_{0}^{t}\left(a X_{s}^{a-1}-\theta_{a} X_{s}^{a}\right) \mathrm{d} s\right)=x^{a} .
$$

Since $\mathrm{E}_{x} X_{t}^{a}<\infty$ for $a \geq 0$, it follows from this formula that $\mathrm{E}_{x} X_{t}^{a-1}<\infty$ for $a>-1$. Once $\mathrm{E}_{x} X_{t}^{a}<\infty$ is established for $a \in(-1,0)$, induction leads to $\mathrm{E}_{x} X_{t}^{a}<\infty$ for $a>a_{\min }$ and $-a \notin \mathbb{N} \cup\{0\}$. If $a \leq a_{\text {min }}$ then (8) is not guaranteed since $p_{a}$ may not be a member of the domain of $\mathcal{A}$.

Letting $f_{a}(t)=\mathrm{E}_{x}\left(X_{t}^{a}\right)$, differentiation yields $f_{a}^{\prime}(t)+\theta_{a} f_{a}(t)=a f_{a-1}(t)$. Applying Laplace transforms then leads to

$$
u \mu_{x}^{a}(u)-f_{a}(0)+\theta_{a} \mu_{x}^{a}(u)=a \mu_{x}^{a-1}(u),
$$

so that (7) follows from the initial condition $f_{a}(0)=x^{a}$.

By Lemma 2, the problem of determining the Laplace transform (6) reduces to finding the solution of an inhomogeneous linear difference equation.

Theorem 3. If $a>a_{\min }$ and $-a \notin \mathbb{N} \cup\{0\}$ then

$$
\begin{aligned}
\mu_{x}^{a}(u)= & \frac{\Gamma(a+1)}{(\lambda+u)^{a}} \prod_{k=1}^{\infty} \frac{\theta_{a+k}+u}{\theta_{k}+u}\left(\frac{1}{u}+\sum_{m=1}^{\infty} \frac{x^{m}}{m !} \prod_{j=1}^{m-1}\left(\theta_{j}+u\right)\right) \\
& -\sum_{k=1}^{\infty} \frac{\Gamma(a+1) x^{a+k}}{\Gamma(a+1+k)} \prod_{j=1}^{k-1}\left(\theta_{a+j}+u\right) .
\end{aligned}
$$

Proof. According to (7), we have to solve the difference equation

$$
\mu^{a}(u)=\frac{\theta_{a+1}+u}{a+1} \mu^{a+1}(u)-\frac{x^{a+1}}{a+1}
$$

with initial condition $\mu^{0}(u)=1 / u$. It follows from the theory of difference equations that the general solution is of the form

$$
\mu^{a}(u)=\omega(a, u) \mu_{\mathrm{H}}^{a}(u)+\mu_{\mathrm{P}}^{a}(u),
$$

where $\mu_{\mathrm{H}}^{a}(u)$ is a solution of the homogeneous equation

$$
\mu_{\mathrm{H}}^{a}(u)=\frac{\theta_{a+1}+u}{a+1} \mu_{\mathrm{H}}^{a+1}(u)
$$

$\mu_{\mathrm{P}}^{a}(u)$ is a particular solution of $(10)$, and $\omega$ is an arbitrary periodic function with $\omega(a, u)=$ $\omega(a+1, u)($ see $[15])$. 
First we try to find a solution of the homogeneous equation. Since the product $\prod_{k=1}^{\infty}\left(\theta_{a+k}+\right.$ $u) /(a+k)$ is 0 , we cannot just repeatedly apply (11). Instead, we let

$$
\hat{\mu}^{a}(u)=\frac{\prod_{k=1}^{\lfloor a\rfloor}\left(\theta_{k}+u\right)}{\Gamma(a+1)} \mu_{\mathrm{H}}^{a}(u),
$$

where $\lfloor a\rfloor=\max \{n \in \mathbb{N} \mid n \leq a\}$. This sequence fulfills the simpler difference equation

$$
\hat{\mu}^{a}(u)=\frac{\theta_{a+1}+u}{\theta_{[a+1]}+u} \hat{\mu}^{a+1}(u) .
$$

In what follows we let $C(u)=\lim _{a \rightarrow \infty} \hat{\mu}^{a}(u)$. Repeated application of (12) yields

$$
\hat{\mu}^{a}(u)=C(u) \prod_{k=1}^{\infty} \frac{\theta_{a+k}+u}{\theta_{[a+k]}+u},
$$

and, hence,

$$
\begin{aligned}
\mu_{\mathrm{H}}^{a}(u) & =C(u) \frac{\Gamma(a+1)}{\prod_{k=1}^{\lfloor a\rfloor}\left(\theta_{k}+u\right)} \prod_{k=1}^{\infty} \frac{\theta_{a+k}+u}{\theta_{[a+k]}+u} \\
& =C(u) \Gamma(a+1) \lim _{N \rightarrow \infty} \frac{\prod_{k=1}^{N} \theta_{a+k}+u}{\prod_{k=1}^{N+\lfloor a\rfloor} \theta_{k}+u} \\
& =C(u) \frac{\Gamma(a+1)}{(\lambda+u)^{\lfloor a\rfloor}} \prod_{k=1}^{\infty} \frac{\theta_{a+k}+u}{\theta_{k}+u} .
\end{aligned}
$$

Since we may multiply the homogeneous solution arbitrarily by any periodic function, we divide by $C(u)(\lambda+u)^{a-\lfloor a\rfloor}$, the reason for this will be given later. We arrive at

$$
\mu_{\mathrm{H}}^{a}(u)=\frac{\Gamma(a+1)}{(\lambda+u)^{a}} \prod_{k=1}^{\infty} \frac{\theta_{a+k}+u}{\theta_{k}+u} .
$$

Next we search for a particular solution of the inhomogeneous equation. This time the repeated application of (10) yields a valid particular solution:

$$
\mu_{\mathrm{P}}^{a}(u)=-\sum_{k=1}^{\infty} \frac{\Gamma(a+1)}{\Gamma(a+1+k)} \prod_{j=1}^{k-1}\left(\theta_{a+j}+u\right) x^{a+k},
$$

as can be checked by inserting $\mu_{\mathrm{P}}^{a}(u)$ into (10).

The final step consists of determining the periodic function $\omega(a, u)$. We adopt ideas from the inventive proof of Proposition 7 of [13] (see also [14]) to show that $\omega(a, u)=\omega(0, u)$.

Recall that a function $f:(0, \infty) \rightarrow(0, \infty)$ is $\log$-convex if $\log f$ is convex. The class of log-convex functions is closed under multiplication, addition, and pointwise limits (see [19]). Bohr-Mollerup's theorem states that a log-convex function $f:(0, \infty) \rightarrow(0, \infty)$ with $f(x+1)=x f(x)$ and $f(1)=1$ must be equal to the gamma function. We show that

$$
G(a)=\Gamma(a) \frac{\omega(a-1, u)}{\omega(0, u)}
$$


is log-convex, so that $\omega(a, u)=\omega(0, u)$. Note that

$$
G(a)=\frac{1}{\omega(0, u)}(\gamma(a)+h(a)),
$$

where

$$
\begin{gathered}
\gamma(a)=\Gamma(a) \frac{\mu^{a-1}(u)}{\mu_{\mathrm{H}}^{a-1}(u)}=\prod_{k=1}^{\infty}\left(\theta_{k}+u\right) \frac{(\lambda+u)^{a-1}}{\prod_{k=1}^{\infty}\left(\theta_{a-1+k}+u\right)} \mu^{a-1}(u), \\
h(a)=-\Gamma(a) \frac{\mu_{\mathrm{P}}^{a-1}(u)}{\mu_{\mathrm{H}}^{a-1}(u)}=\prod_{k=1}^{\infty}\left(\theta_{k}+u\right) \sum_{k=1}^{\infty} \frac{\Gamma(a)}{\Gamma(a+k)} \frac{(\lambda+u)^{a-1} x^{a-1+k}}{\prod_{j=k}^{\infty}\left(\theta_{a-1+j}+u\right)} .
\end{gathered}
$$

Since $\mu^{a-1}(u)$ is the Laplace transform of the log-convex function $a \mapsto \mathrm{E}_{x} X_{t}^{a}$ (see [14]), it follows that $a \mapsto \mu^{a-1}(u)$ is also log-convex. The function $a \mapsto(\lambda+u)^{a-1}$ is obviously log-convex, this is in fact the reason why we chose $(\lambda+u)^{a}$ instead of $(\lambda+u)^{\lfloor a\rfloor}$ in (13). The function $a \mapsto \theta_{a-1+k}+u$ is log-concave since $a \mapsto \mathrm{E}_{x} Q^{a+k}$ is convex. Thus, $a \mapsto$ $\left(\prod_{k=1}^{\infty}\left(\theta_{a-1+k}+u\right)\right)^{-1}$ is log-convex and it follows that $\gamma(a)$ is log-convex.

To show log-convexity of $h$, note that

$$
\frac{\Gamma(a)}{\Gamma(a+k)}=\frac{1}{(a+1)(a+2) \cdots(a+k-1)}
$$

is $\log$-convex since $a \mapsto \log a$ is concave. Log-convexity of the sum in $h$ follows as before. We have thus shown that $G$ is $\log$-convex. Since $G(a+1)=a G(a)$ and $G(1)=1$, it follows from Bohr-Mollerup's theorem that $G(a)=\Gamma(a)$ and, thus, $\omega(a-1, u)=\omega(0, u)$. It immediately follows from the initial condition $\mu^{0}(u)=1 / u$ that

$$
\omega(0, u)=1+u \sum_{k=1}^{\infty} \frac{x^{k}}{k !} \prod_{j=1}^{k-1}\left(\theta_{j}+u\right)
$$

which completes the proof.

Note that $\mu_{x}^{a}(u)=(1 / u) \mathrm{E}_{x}\left(X_{Z}^{a}\right)$, where $Z$ is some independent, exponentially distributed random variable with mean $1 / u$. If we multiply $\mu_{x}^{a}(u)$ by $u$ and let $u$ tend to 0 , we obtain the following corollary, identifying the fractional stationary moments.

Corollary 1. If $a>a_{\min }$ and $-a \notin \mathbb{N} \cup\{0\}$ then the fractional moments of the limiting distribution are given by

$$
\mathrm{E}\left(X_{\infty}^{a}\right)=\frac{\Gamma(a+1)}{\lambda^{a}} \prod_{k=1}^{\infty} \frac{\theta_{a+k}}{\theta_{k}} .
$$

A simple calculation leads to the following result, which can be found in [6] for the $x=0$ case.

Corollary 2. For integer values $a=n \in \mathbb{N}$, (9) reduces to

$$
\mu_{x}^{n}(u)=\frac{n !}{\prod_{k=1}^{n}\left(\theta_{k}+u\right)}\left(\frac{1}{u}+\sum_{k=1}^{n} \frac{x^{k}}{k !} \prod_{j=1}^{k-1}\left(\theta_{j}+u\right)\right) .
$$


An inversion of (14) is possible and results in Theorem 4, below. It provides the precise rate of convergence of $\mathrm{E}_{x}\left(X_{t}^{n}\right)$ to the stationary limit $\mathrm{E}\left(X_{\infty}^{n}\right)$ in terms of the exponential functions $\exp \left(-\theta_{m} t\right)$.

Theorem 4. If $n \in \mathbb{N}$, the nth transient moment of $X_{t}$ is

$$
\mathrm{E}_{x}\left(X_{t}^{n}\right)=\frac{n !}{\prod_{i=1}^{n} \theta_{i}}+n ! \sum_{m=1}^{n}\left(\sum_{k=0}^{m} \frac{x^{k}}{k !} \prod_{j=k, j \neq m}^{n} \frac{1}{\left(\theta_{j}-\theta_{m}\right)}\right) \exp \left(-\theta_{m} t\right) .
$$

Proof. By partial fraction expansion we obtain

$$
\frac{1}{\prod_{j=k}^{n}\left(\theta_{j}+u\right)}=\sum_{m=k}^{n} \frac{1}{\left(\theta_{m}+u\right) \prod_{j=k, j \neq m}^{n}\left(\theta_{j}-\theta_{m}\right)}
$$

so that we can write (14) as

$$
\frac{\mu_{x}^{n}(u)}{n !}=\sum_{m=1}^{n} \frac{1}{\theta_{m}+u}\left(\frac{1}{u} \frac{1}{\prod_{j=1, j \neq m}^{n}\left(\theta_{j}-\theta_{m}\right)}+\sum_{k=1}^{m} \frac{x^{k}}{k !} \frac{1}{\prod_{j=k, j \neq m}^{n}\left(\theta_{j}-\theta_{m}\right)}\right) .
$$

Since $1 /\left(\theta_{m}+u\right)$ is the Laplace transform of $\exp \left(-\theta_{m} t\right)$ and $(1 / u)\left(1 /\left(\theta_{m}+u\right)\right)$ is the transform of $-\exp \left(-\theta_{m} t\right) / \theta_{m}$, we have

$$
\begin{aligned}
\frac{\mathrm{E}_{x}\left(X_{t}^{n}\right)}{n !} & =\sum_{m=1}^{n}\left(\frac{1-\exp \left(-\theta_{m} t\right)}{\theta_{m} \prod_{j=1, j \neq m}^{n}\left(\theta_{j}-\theta_{m}\right)}+\sum_{k=1}^{m} \frac{x^{k}}{k !} \frac{\exp \left(-\theta_{m} t\right)}{\prod_{j=k, j \neq m}^{n}\left(\theta_{j}-\theta_{m}\right)}\right) \\
& =\sum_{m=1}^{n}\left(\frac{1}{\theta_{m} \prod_{j=1, j \neq m}^{n}\left(\theta_{j}-\theta_{m}\right)}+\left(\sum_{k=0}^{m} \frac{x^{k}}{k ! \prod_{j=k, j \neq m}^{n}\left(\theta_{j}-\theta_{m}\right)}\right) \exp \left(-\theta_{m} t\right)\right) .
\end{aligned}
$$

A further application of the partial fraction expansion yields (15).

We remark that (15) may also be written as a polynomial in $x$ :

$$
\mathrm{E}_{x}\left(X_{t}^{n}\right)=n ! \sum_{k=0}^{n} \frac{x^{k}}{k !} \sum_{m=k}^{n} \prod_{j=k, j \neq m}^{n} \frac{\exp \left(-\theta_{m} t\right)}{\theta_{j}-\theta_{m}} .
$$

Ott and Kemperman [17] derived, for the case in which $Q=q$, the expression

$$
\begin{aligned}
\mathrm{E}_{x}\left(X_{t}^{n}\right)= & \frac{n !}{\lambda^{n}} \sum_{k=0}^{n} \frac{\lambda^{k} x^{k}}{k !} q^{-k(n-k)} \\
& \times\left(\sum_{m=k}^{n} \frac{(-1)^{m-k} q^{(1 / 2)(m-k)(m-k-1)-(m-k)(n-k-1)}}{(1-q) \cdots\left(1-q^{n-m}\right)(1-q) \cdots\left(1-q^{m-k}\right)} \exp \left(-\lambda t\left(1-q^{m}\right)\right)\right),
\end{aligned}
$$

which can indeed be shown to be equal to (15). For the same case, Ott and Kemperman [17] derived expressions for the fractional transient moments through an explicit characterization of the transient distribution. 


\section{Discussion}

Formula (15) leads to

$$
\mathrm{E}_{x} X_{t}=\frac{1}{\theta_{1}}-\frac{\left(1-\theta_{1} x\right) \exp \left(-\theta_{1} t\right)}{\theta_{1}},
$$

which shows exponential convergence to the stationary mean $\mathrm{E} X_{\infty}=1 / \theta_{1}$. If the relaxation time is defined as

$$
r_{x}(\varepsilon)=\inf \left\{t \geq 0:\left|1-\frac{\mathrm{E}_{x} X_{t}}{\mathrm{E} X_{\infty}}\right|<\varepsilon\right\},
$$

the following result follows immediately from (16).

Corollary 3. We have

$$
r_{x}(\varepsilon)=\frac{1}{\theta_{1}} \log \frac{\left|1-\theta_{1} x\right|}{\varepsilon}, \quad \varepsilon<\left|\frac{1}{\theta_{1}}-x\right| .
$$

A further measure for the speed of convergence to the steady state may follow from the formula for the variance, which is given by

$$
\begin{aligned}
\operatorname{var}_{x}\left(X_{t}\right)= & \frac{2 \theta_{1}-\theta_{2}}{\theta_{1}^{2} \theta_{2}}+2\left(\frac{2 \theta_{1}-\theta_{2}}{\theta_{1}^{2}\left(\theta_{1}-\theta_{2}\right)}-\frac{2 \theta_{1}-\theta_{2}}{\theta_{1}\left(\theta_{1}-\theta_{2}\right)} x\right) \exp \left(-\theta_{1} t\right) \\
& -\left(\frac{2}{\theta_{2}\left(\theta_{1}-\theta_{2}\right)}-\frac{2}{\theta_{1}-\theta_{2}} x-x^{2}\right) \exp \left(-\theta_{2} t\right) \\
& -\left(\frac{1}{\theta_{1}^{2}}-\frac{2}{\theta_{1}} x+x^{2}\right) \exp \left(-2 \theta_{1} t\right) .
\end{aligned}
$$

Note that the exponential terms in (17) appear according to their magnitude, since

$$
\theta_{1} \leq \theta_{2}=\lambda\left(1-\mathrm{E}\left(Q^{2}\right)\right) \leq \lambda\left(1-(\mathrm{E} Q)^{2}\right)=\lambda(1-\mathrm{E} Q)(1+\mathrm{E} Q) \leq 2 \theta_{1} .
$$

If we start the process in $x=\mathrm{E} X_{\infty}=1 / \theta_{1}$ then (17) reduces to the simple expression

$$
\operatorname{var}_{x}\left(X_{t}\right)=\operatorname{var} X_{\infty}\left(1-\exp \left(-\theta_{2} t\right)\right) \text {. }
$$

For the case in which $x=0$ and $Q \stackrel{\mathrm{D}}{=} U$, with $U$ a uniformly distributed random variable on $[0,1)$, Theorems 4 and 5 of [7] coincide with (16) and (17), respectively. For the case in which $Q=q,(16)$ and (17) agree with (9.1) and (9.2) of [16].

\section{Connection to Lévy processes}

The transformation $X_{t} \mapsto \log X_{t}$ converts the multiplicative jumps of the process $X_{t}$ into additive jumps with i.i.d. sizes. Let

$$
L_{t}=-\log W_{t}=-\sum_{k=1}^{N_{t}} \log Q_{k}
$$

be the associated compound Poisson process. Then the process $Y_{t}=X_{t} \exp \left(L_{t}\right)$ has absolutely continuous paths. Moreover, $\exp \left(L_{t}\right)$ is piecewise constant and $X_{t}$ has slope 1 in between the jumps, so that the density of $Y_{t}$ is given by $Y_{t}^{\prime}=\exp \left(L_{t}\right) X_{t}^{\prime}+0=\exp \left(L_{t}\right)$. Hence,

$$
X_{t}=\exp \left(-L_{t}\right) X_{0}+\int_{0}^{t} \exp \left(L_{s}-L_{t}\right) \mathrm{d} s \stackrel{\mathrm{D}}{=} \exp \left(-L_{t}\right) X_{0}+\int_{0}^{t} \exp \left(L_{s-t}\right) \mathrm{d} s,
$$


because $L_{t}$ has stationary increments and $X_{0}=Y_{0}$. It follows that

$$
X_{t} \stackrel{\mathrm{D}}{=} \exp \left(-L_{t}\right) X_{0}+\int_{0}^{t} \exp \left(-L_{s}\right) \mathrm{d} s .
$$

The function $a \mapsto \theta_{a}$ is the Laplace exponent of the Lévy process $L_{t}$, since

$$
\theta_{a}=\lambda\left(1-\int_{0}^{1} q^{a} \mathrm{~d} H(q)\right)=\lambda\left(1-\int_{0}^{\infty} \mathrm{e}^{-u a} \mathrm{~d} H\left(\mathrm{e}^{-u}\right)\right)=\lambda(1-\beta(a)),
$$

where $\beta(a)$ is the Laplace transform of $-\log Q$.

For $t$ tending to $\infty$, it is readily seen from (18) that

$$
X_{\infty} \stackrel{\mathrm{D}}{=} \int_{0}^{\infty} \exp \left(-L_{s}\right) \mathrm{d} s
$$

which relates the stationary distribution of the Markov process $\left(X_{t}\right)_{t \geq 0}$ to the terminating value of the exponential functional. This relation has already been observed in [13, Section 3] (see also [8]).

\section{References}

[1] Altman, E., Avrachenkov, K. E., Barakat, C. and Núñez QueiJa, R. (2002). State-dependent M/G/1 type queueing analysis for congestion control in data networks. Comput. Networks 39, 789-808.

[2] Altman, E., Avrachenkov, K. E., Kherani, A. A. and Prabhu, B. J. (2005). Performance analysis and stochastic stability of congestion control protocols. In Proc. IEEE INFOCOM.

[3] Asmussen, S. (2003). Applied Probability and Queues. Springer, New York.

[4] BaCCELli, F. AND McDonald, D. R. (2006). Mellin transforms for TCP throughput with applications to cross layer optimization. In Proc. 40th Annual Conf. Inf. Sci. Systems, IEEE, pp. 32-37.

[5] Baccelli, F., Kim, K. B. AND McDonald, D. R. (2007). Equilibria of a class of transport equations arising in congestion control. Queueing Systems 55, 1-8.

[6] Bertoin, J. And Yor, M. (2005). Exponential functionals of Lévy processes. Prob. Surveys 2, 191-212.

[7] Boxma, O., Perry, D., Stadje, W. and Zacks, S. (2006). A Markovian growth-collapse model. Adv. Appl. Prob. 38, 221-243.

[8] Carmona, P., Petit, F. and Yor, M. (2001). Exponential functionals of Lévy processes. In Lévy Processes, eds O. Barndorff-Nielsen et al., Birkhäuser, Boston, MA, pp. 41-55.

[9] Davis, M. (1993). Markov Models and Optimization. Chapman \& Hall, London.

[10] EliazAR, I. AND KLAFTER, K. (2004). A growth-collapse model: Lévy inflow, geometric crashes, and generalized Ornstein-Uhlenbeck dynamics. Physica A 334, 1-21.

[11] Guessing, H. and Paulsen, J. (1997). Present value distributions with application to ruin theory and stochastic equations. Stoch. Process. Appl. 71, 123-144.

[12] Gnedin, A., Pitman, J. And Yor, M. (2006). Asymptotic laws for compositions derived from transformed subordinators. Ann. Prob. 34, 468-492.

[13] Guillemin, F., Robert, P. and Zwart, B. (2004). AIMD algorithms and exponential functionals. Ann. Appl. Prob. 14, 90-117.

[14] Maulik, K. and Zwart, B. (2006). Tail asymptotics for exponential functionals of Lévy processes. Stoch. Process. Appl. 116, 156-177.

[15] Milne-Thomson, L. M. (1933). The Calculus of Finite Differences. Macmillan, London.

[16] Отт, T. J. (2005). Transport protocols in the TCP paradigm and their performance. Telecommun. Systems 30, 351-385.

[17] OtT, T. J. And Kemperman, J. H. B. (2007). The transient behavior of processes in the TCP paradigm. To appear in Prob. Eng. Inf. Sci.

[18] Ott, T. J., Kemperman, J. H. B. And Mathis, M. (1996). The stationary behavior of ideal TCP congestion avoidance. Unpublished manuscript. Available at www.teunisott.com.

[19] Roberts, A. W. and Varberg, D. E. (1973). Convex Functions. Academic Press, New York. 
[20] Rolski, T., Schmidli, H., Schmidt, V. And Teugels, J. (1999). Stochastic Processes for Insurance and Finance. John Wiley, New York.

[21] Shanthikumar, J. And Sumita, U. (1983). General shock models associated with correlated renewal sequences. J. Appl. Prob. 20, 600-614.

[22] VervaAt, W. (1979). On a stochastic difference equation and a representation of non-negative infinitely divisible random variables. Adv. Appl. Prob. 11, 750-783. 